\title{
Decomposition of Bipartite Graphs under Degree Constraints
}

\section{H. J. Broersma}

Department of Applied Mathematics, University of Twente, P.O. Box 217, 7500 AE Enschede. The Netherlands

\section{R. J. Faudree}

Department of Mathematical Sciences, Memphis State University, Memphis, Tennessee 38152

\section{J. van den Heuvel and H. J. Veldman}

Department of Applied Mathematics, University of Twente, P.O. Box 217, 7500 AE Enschede, The Netherlands

Let $G=(A, B ; E)$ be a bipartite graph. Let $e_{1}, e_{2}$ be nonnegative integers, and $t_{1}, t_{2}$ nonnegative integer-valued functions on $V(G)$ such that $e_{i} \leq|E| \leq \theta_{1}+e_{2}$ and $f_{i}(v) \leq d(v) \leq f_{1}(v)+f_{2}(v)$ for all $v \in$ $V(G)(i=1,2)$. Necessary and sufficient conditions are obtained for $G$ to admit a decomposition in spanning subgraphs $G_{1}=\left(A, B ; E_{1}\right)$ and $G_{2}=\left(A, B ; E_{2}\right)$ such that $\left|E_{i}\right| \leq e_{i}$ and $d_{G_{i}}(v) \leq f_{i}(v)$ for all $v$ $\in V(G)(i=1,2)$. The result generalizes a known characterization of bipartite graphs with a $k$-factor. Its proof uses flow theory and is a refinement of the proof of an analogous result due to Folkman and Fulkerson. By applying corresponding flow algorithms, the described decomposition can be found in polynomial time if it exists. As an application, an assignment problem is solved. (c) 1993 by John Wiley \& Sons, Inc.

\section{AN ASSIGNMENT PROBLEM}

The following problem was posed in van Doorn [6]. In a certain year, each of $k$ students at some school is required to spend probationary periods $p_{1}, \ldots, p_{m}$ at $m$ different companies from a collection $\left\{c_{1}, \ldots, c_{n}\right\}$ of $n$ companies. In the period $p_{i}$, company $c_{j}$ can host at most $r_{i j}$ students $(i=1, \ldots, m ; j=1, \ldots, n)$. The question is whether and how the probation requirements can be satisfied for all students.

After introducing suitable notation and terminology in Section 2, we present in Section 3 a solution of this problem using the theory of $k$-factors in bipartite graphs. In Section 4, the results in Section 3 are gener- alized to an analog of a theorem due to Folkman and Fulkerson [2]. Using this generalization, another solution of the problem, not using $k$-factors, is presented in Section 5 .

\section{NOTATION AND TERMINOLOGY}

We use Bondy and Murty [1] for notation and terminology not defined here. The graphs we consider may have multiple edges, but no loops.

Let $G$ be a bipartite graph with bipartition $(A, B)$. We write $G=(A, B)$, or $G=(A, B ; E)$ if $G$ has edge set $E$. The graph $G$ is balanced if $|A|=|B|$. For $X \subseteq A$ and 
$Y \subseteq B, q_{G}(X, Y)$ or $q(X, Y)$ denotes the number of edges with one end in $X$ and the other in $Y$. For $y \in Y$, we write $q(X, y)$ instead of $q(X,\{y\})$. If $M$ is a matching in $G$, then $M$ is $S$-saturating if every vertex of $S$ is incident with an edge of $M$. For a nonnegative integer $k$, a $k$-factor of $G$ is a $k$-regular spanning subgraph of $G$. More generally, for a nonnegative integer-valued function $f$ on $V(G)$, an $f$-factor is a spanning subgraph $G^{\prime}$ of $G$ such that $d_{G^{\prime}}(v)=f(v)$ for all $v \in V(G)$. The function $f$ is balanced if $\Sigma_{x \in A} f(x)=\Sigma_{y \in B} f(y)$.

\section{SOLUTION OF THE PROBLEM USING $k$-FACTORS}

Consider the following question:

(1) Given a bipartite graph $G=(A, B)$ and a nonnegative integer $k$, do there exist $k$ pairwise disjoint $A$ saturating matchings?

As shown in van Doorn [6], the assignment problem of Section 1 can be translated into (1) by defining a bipartite graph $G=(A, B)$ with $A=\left\{p_{1}, \ldots, p_{m}\right\}$ and $B=$ $\left\{c_{1}, \ldots, c_{n}\right\}$ in which the vertices $p_{i}$ and $c_{j}$ are joined by $r_{i j}$ edges $(i=1, \ldots, m ; j=1, \ldots, n)$. The probation requirements can be satisfied for all $k$ students if and only if $G$ admits $k$ pairwise disjoint $A$ saturating matchings.

A necessary and sufficient condition for (1) to have an affirmative answer can be derived from the following result on $f$-factors, attributed in Katerinis [4] to Ore and Ryser.

Theorem 1. (Ore-Ryser $f$-factor theorem, see [4]). Let $G=(A, B)$ be a bipartite graph and $f$ a balanced nonnegative integer-valued function on $V(G)$. Then, $G$ has an $f$-factor if and only if, for all $X \subseteq A$,

$$
\sum_{r \in B} \min \{f(y), q(X, y)\} \geq \sum_{x \in X} f(x) .
$$

An immediate consequence of Theorem 1 is the following:

Corollary 2. Let $G=(A, B)$ be a balanced bipartite graph and $k$ a nonnegative integer. Then, $G$ has a $k$ factor if and only if, for all $X \subseteq A$,

$$
\sum_{y \in B} \min \{k, q(X, y)\} \geq k|X| .
$$

Now the following consequence of Corollary 2 can be used to answer question (1):
Corollary 3. Let $G=(A, B)$ be a bipartite graph, and $k$ a nonnegative integer. Then, $G$ has $k$ pairwise disjoint $A$-saturating matchings if and only if, for all $X \subseteq A$,

$$
\sum_{y \in B} \min \{k, q(X, y)\} \geq k|X| .
$$

Proof. We may assume $|A| \leq|B|$. Construct a balanced bipartite graph $G^{*}=\left(A^{*}, B\right)$ from $G=(A, B)$ by adding $|B|-|A|$ new vertices, each of which is joined by $k$ edges to each vertex of $B$. Then, (4) holds for all $X \subseteq A$ if and only if, for all $X^{*} \subseteq A^{*}$,

$$
\sum_{y \in B} \min \left\{k, q_{G^{*}}\left(X^{*}, y\right)\right\} \geq k\left|X^{*}\right| .
$$

(Note that if $X^{*}$ contains a vertex of $A^{*}-A$, then the left-hand side of (5) equals $k|B|$.) We now show that $G$ has $k$ pairwise disjoint $A$-saturating matchings if and only if $G^{*}$ has a $k$-factor; hence, Corollary 3 follows from Corollary 2. Clearly, if $G$ has $k$ pairwise disjoint $A$-saturating matchings, then $G^{*}$ has $k$ pairwise disjoint perfect matchings and, hence, a $k$-factor. Conversely, if $G^{*}$ has a $k$-factor $H^{*}$, then $G$ has a spanning subgraph $H$ such that $d_{H}(x)=k$ for all $x \in A$ and $d_{H}(y)$ $\leq k$ for all $y \in B$. By $[1$, Theorem 6.1], $H$ admits a $k$ edge-coloring. The color classes associated with such a coloring constitute $k$ pairwise disjoint $A$-saturating matchings of $G$.

Note that for $k=1$, Corollary 3 reduces to Hall's Theorem [1, Theorem 5.2].

Theorem 1 is proved via the max-flow min-cut theorem (see Ford and Fulkerson [3]). By using the associated labeling method in [3], question (1) can be answered in polynomial time. Moreover, a collection of $k$ pairwise disjoint $A$-saturating matchings in a bipartite graph $G=(A, B)$ can actually be constructed in polynomial time if it exists.

\section{A GENERALIZATION OF THE ORE-RYSER f-FACTOR THEOREM}

Our main result is the following:

Theorem 4. Let $G=(A, B ; E)$ be a bipartite graph with $e=|E|$. Let $f_{1}, f_{2}$ be nonnegative integer-valued functions on $V(G)$, and $e_{1}, e_{2}$ nonnegative integers satisfying

$$
\begin{array}{ll}
f_{i}(v) \leq d(v) \leq f_{1}(v)+f_{2}(v) & (v \in V(G), i=1,2), \\
e_{i} \leq e \leq e_{1}+e_{2} & (i=1,2) .
\end{array}
$$


Then, there exist spanning subgraphs $G_{1}=\left(A, B ; E_{1}\right)$ and $G_{2}=\left(A, B ; E_{2}\right)$ of $G$ satisfying

$$
\begin{array}{ll}
E_{1} \cup E_{2}=E, E_{1} \cap E_{2}=\varnothing, \\
\left|E_{i}\right| \leq e_{i} & (i=1,2), \\
d_{G_{1}}(v) \leq f_{i}(v) & (v \in V(G), i=1,2)
\end{array}
$$

if and only if, for all $X \subseteq A$,

$$
\begin{aligned}
& \sum_{y \in B} \min \left\{f_{1}(y), q(X, y)\right\} \geq \sum_{x \in X}\left(d(x)-f_{2}(x)\right), \\
& \sum_{y \in B} \min \left\{f_{1}(y), q(X, y)\right\} \geq e-e_{2}-\sum_{x \in A-X} f_{1}(x), \\
& \sum_{y \in B} \min \left\{f_{2}(y), q(X, y)\right\} \geq \sum_{x \in X}\left(d(x)-f_{1}(x)\right), \\
& \sum_{y \in B} \min \left\{f_{2}(y), q(X, y)\right\} \geq e-e_{1}-\sum_{x \in A-X} f_{2}(x) .
\end{aligned}
$$

Proof. Suppose (6) holds for spanning subgraphs $G_{1}=\left(A, B ; E_{1}\right)$ and $G_{2}=\left(A, B ; E_{2}\right)$ of $G$, and let $X \subseteq$ $A$. Then,

$$
\begin{aligned}
q_{G_{1}}(X, B) & =q_{G}(X, B)-q_{G_{2}}(X, B) \\
& =\sum_{x \in X} d_{G}(x)-\sum_{x \in X} d_{G_{2}}(x) \geq \sum_{x \in X}\left(d_{G}(x)-f_{2}(x)\right)
\end{aligned}
$$

Also,

$$
\begin{aligned}
q_{G_{1}}(X, B) & =\left|E_{1}\right|-\sum_{x \in A-X} d_{G_{1}}(x) \\
& =e-\left|E_{2}\right|-\sum_{x \in A-X} d_{G_{1}}(x) \geq e-e_{2}-\sum_{x \in A-X} f_{1}(x) .
\end{aligned}
$$

On the other hand,

$$
\begin{aligned}
q_{G_{1}}(X, B) & =\sum_{y \in B} q_{G_{1}}(X, y) \leq \sum_{y \in B} \min \left\{d_{G_{1}}(y), q_{G}(X, y)\right\} \\
& \leq \sum_{y \in B} \min \left\{f_{1}(y), q_{G}(X, y)\right\} .
\end{aligned}
$$

It follows that (7) and (8) hold. Similarly, (9) and (10) hold.

Conversely, assume that (7)-(10) hold. We construct a network $G^{\prime}$ from $G$ by orienting all edges of $G$ from $A$ to $B$ and adding two new vertices $a$ and $b$ together with the edges $(b, a),(a, x)$ for each $x \in A$, and $(y, b)$ for each $y \in B$. We define nonnegative integer-valued functions $l$ and $c$ on $E\left(G^{\prime}\right)$ as follows: $l(a, x)=d_{G}(x)-f_{2}(x), c(a, x)=f_{1}(x) \quad(x \in A)$,

$l(x, y)=0, c(x, y)=1$

$\left(x \in A, y \in B,(x, y) \in E\left(G^{\prime}\right)\right)$,

$l(y, b)=d_{G}(y)-f_{2}(y), c(y, b)=f_{1}(y) \quad(y \in B)$,

$l(b, a)=e-e_{2}, c(b, a)=e_{1}$.

An integer-valued function $f$ on the edge set of $G^{\prime}$ is called a feasible flow if Kirchhoff's law is satisfied at each vertex of $G^{\prime}$ and $l(u, v) \leq f(u, v) \leq c(u, v)$ for each edge $(u, v)$ of $G^{\prime}$. If $f$ is a feasible flow, then the spanning subgraphs $G_{1}=\left(A, B ; E_{1}\right)$ and $G_{2}=\left(A, B ; E_{2}\right)$ of $G$ with $E_{1}=\{(x, y) \in E \mid f(x, y)=1\}$ and $E_{2}=\{(x, y) \in$ $E \mid f(x, y)=0\}$, respectively, satisfy (6). (For example, $\left|E_{2}\right|=e-\left|E_{1}\right|=e-\Sigma_{(x, y) \in E_{1}} f(x, y)=e-\sum_{x \in A} f(a, x)=$ $e-f(b, a) \leq e-l(b, a)=e_{2}$ and, for $x \in A, d_{G_{1}}(x)=$ $f(a, x) \leq c(a, x)=f_{1}(x)$.) We will establish the existence of a feasible flow. By [3, Theorem 3.1], a feasible flow exists if and only if, for all $Z \subseteq V\left(G^{\prime}\right)$,

$$
\sum_{\substack{u \in Z \\ v \in \bar{Z}}} c(u, v) \geq \sum_{\substack{u \in \bar{Z} \\ v \in \bar{Z}}} l(u, v)
$$

where $\bar{Z}=V\left(G^{\prime}\right)-Z$.

Let $Z \subseteq V\left(G^{\prime}\right), X=Z \cap A, Y=Z \cap B$. If $a, b \in \bar{Z}$, then by (7),

$$
\begin{aligned}
\sum_{\substack{u \in Z \\
v \in \bar{Z}}} c(u, v) & =\sum_{y \in Y} f_{1}(y)+q_{G}(X, B-Y) \\
& =\sum_{y \in Y} f_{1}(y)+\sum_{y \in B-Y} q_{G}(X, y) \\
& \geq \sum_{y \in B} \min \left\{f_{1}(y), q_{G}(X, y)\right\} \\
& \geq \sum_{x \in X}\left(d_{G}(x)-f_{2}(x)\right)=\sum_{\substack{u \in \bar{Z} \\
v \in Z}} l(u, v) .
\end{aligned}
$$

If $a \in Z, b \in \bar{Z}$, then by (8),

$$
\begin{aligned}
\sum_{\substack{u \in \bar{Z} \\
v \in \bar{Z}}} c(u, v) & =\sum_{y \in Y} f_{1}(y)+q_{G}(X, B-Y)+\sum_{x \in A-X} f_{1}(x) \\
& \geq \sum_{y \in B} \min \left\{f_{1}(y), q_{G}(X, y)\right\}+\sum_{x \in A-X} f_{1}(x) \\
& \geq\left(e-e_{2}-\sum_{x \in A-X} f_{1}(x)\right)+\sum_{x \in A-X} f_{1}(x) \\
& =e-e_{2}=\sum_{\substack{u \in \bar{Z} \\
v \in Z}} l(u, v) .
\end{aligned}
$$


If $a \in \bar{Z}, b \in Z$, then by (10),

$$
\begin{aligned}
e-e_{1}-\sum_{x \in X} f_{2}(x) \leq & \sum_{y \in B} \min \left\{f_{2}(y), q_{G}(A-X, y)\right\} \\
& \leq \sum_{y \in B-Y} f_{2}(y)+\sum_{y \in Y} q_{G}(A-X, y) \\
& =\sum_{y \in B-Y} f_{2}(y)+q_{G}(A-X, Y) \\
& =\sum_{y \in B-Y} f_{2}(y)+e-\sum_{x \in X} d_{G}(x) \\
& -\sum_{y \in B-Y} d_{G}(y)+q_{G}(X, B-Y) ;
\end{aligned}
$$

hence,

$$
\begin{aligned}
\sum_{\substack{u \in \mathcal{L} \\
v \in \bar{Z}}} c(u, v) & =q_{G}(X, B-Y)+e_{1} \\
& \geq \sum_{x \in X}\left(d_{G}(x)-f_{2}(x)\right)+\sum_{v \in B-\gamma}\left(d_{G}(y)-f_{2}(y)\right) \\
& =\sum_{\substack{u \in \bar{Z} \\
v \in \mathcal{Z}}} l(u, v)
\end{aligned}
$$

Finally, if $a, b \in Z$, then by (9),

$$
\begin{aligned}
\sum_{x \in A-X}\left(d_{G}(x)-f_{1}(x)\right) \leq & \sum_{y \in B} \min \left\{f_{2}(y), q_{G}(A-X, y)\right\} \\
& \leq \sum_{y \in B-Y} f_{2}(y)+\sum_{y \in Y} q_{G}(A-X, y) \\
& =\sum_{y \in B-Y} f_{2}(y)+q_{G}(A-X, Y) \\
& =\sum_{y \in B-Y} f_{2}(y)+\sum_{x \in A-X} d_{G}(x) \\
& -\sum_{y \in H-Y} d_{G}(y)+q_{G}(X, B-Y)
\end{aligned}
$$

hence,

$$
\begin{aligned}
\sum_{\substack{u \in \mathcal{Z} \\
v \in \bar{Z}}} c(u, v) & =\sum_{x \in A-X} f_{1}(x)+q_{G}(X, B-Y) \\
& \geq \sum_{y \in B-Y}\left(d_{G}(y)-f_{2}(y)\right)=\sum_{\substack{u \in \bar{Z} \\
v \in Z}} l(u, v) .
\end{aligned}
$$

Having established (11) for all $Z \subseteq V\left(G^{\prime}\right)$, we are done.

As we show next, Theorem 1 can be obtained from Theorem 4. Set

$$
\begin{aligned}
& f_{1}(v)=f(v) \quad \text { for all } v \in V(G), \\
& f_{2}(v)= \begin{cases}d(v)-f(v) & \text { if } v \in A \\
d(v) & \text { if } v \in B\end{cases} \\
& e_{1}=e_{2}=e .
\end{aligned}
$$

Then (7) amounts to (2), while (8) and (10) are trivially satisfied. The inequality (9), too, is trivially satisfied, since

$$
\begin{aligned}
\sum_{y \in \boldsymbol{B}} \min \{d(y), q(X, y)\} & =\sum_{y \in B} q(X, y)=q(X, B) \\
& =\sum_{x \in X} d(x) \geq \sum_{x \in X}(d(x)-f(x)) .
\end{aligned}
$$

Hence under (2), $G$ contains, by Theorem 4, a spanning subgraph $G_{1}=\left(A, B ; E_{1}\right)$ such that $\left|E_{1}\right|=e_{1}$, $d_{G_{1}}(x)=f(x)$ for all $x \in A$ and $d_{G}(y) \leq f(y)$ for all $y \in$ $B$. Since $\Sigma_{x \in A} d_{G_{1}}(x)=\sum_{y \in B} d_{G_{1}}(y)$ and $\sum_{x \in A} f(x)=\Sigma_{y \in B}$ $f(y)$, in fact, $d_{G_{1}}(y)=f(y)$ for all $y \in B$, implying that $G_{1}$ is an $f$-factor of $G$. Thus, the sufficiency of (2) in Theorem 1 follows from the sufficiency of (7)-(10) in Theorem 4. Similarly, the necessity of (2) in Theorem 1 follows from the necessity of (7)-(10) in Theorem 4.

The proof of Theorem 4 is a refinement of the proof of the following analogous result.

Theorem 5 (Folkman and Fulkerson [2]). Let $G=(A$, $B ; E)$ be a bipartite graph with $e=|E|$. Let $f_{1}, f_{2}$ be nonnegative integer-valued functions on $V(G)$, and $e_{1}$, $e_{2}$ nonnegative integers, satisfying

$$
\begin{aligned}
& d(v) \leq f_{1}(v)+f_{2}(v) \quad(v \in V(G)), \\
& e=e_{1}+e_{2} .
\end{aligned}
$$

Then, there exist spanning subgraphs $G_{1}=\left(A, B ; E_{1}\right)$ and $G_{2}=\left(A, B ; E_{2}\right)$ of $G$ satisfying

$$
\begin{array}{ll}
E_{1} \cup E_{2}=E, E_{1} \cap E_{2}=\varnothing, \\
\left|E_{i}\right|=e_{i} \quad(i=1,2), \\
d_{G_{i}}(v) \leq f_{i}(v) \quad(v \in V(G), i=1,2)
\end{array}
$$

if and only if, for all $X \subseteq A$ and $Y \subseteq B$,

$$
\begin{aligned}
& q(X, Y) \geq e_{1}-\sum_{x \in A-X} f_{1}(x)-\sum_{y \in B-Y} f_{1}(y), \\
& q(X, Y) \geq e_{2}-\sum_{x \in A-X} f_{2}(x)-\sum_{y \in B-Y} f_{2}(y),
\end{aligned}
$$




$$
\begin{aligned}
& q(X, Y) \leq \sum_{x \in X} f_{1}(x)+\sum_{y \in Y} f_{2}(y), \\
& q(X, Y) \leq \sum_{x \in X} f_{2}(x)+\sum_{y \in Y} f_{1}(y) .
\end{aligned}
$$

Note that Theorem 4 is of slightly wider applicability than is Theorem 5 because of the weaker restrictions on $e_{1}$ and $e_{2}$. The condition $f_{i}(v) \leq d(v)(v \in V(G), i=$ 1,2 ), occurring in Theorem 4 but not in Theorem 5 , is no real restriction; it merely serves to give Theorem 4 a nicer shape.

Theorem 5 has consequences analogous to Theorem 1, Corollary 2, and Corollary 3. The analog of Theorem 1 is in Lovász and Plummer [5, Theorem 2.4.2]. Here, we only mention the analog of Corollary 3.

Corollary 6. Let $G=(A, B)$ be a bipartite graph and $k$ a nonnegative integer. Then, $G$ has $k$ pairwise disjoint $A$-saturating matchings if and only if, for all $X \subseteq A$ and $Y \subseteq B$,

$$
q(X, Y) \geq k(|X|-|B-Y|) .
$$

Problem (1) is "one-sided" in the sense that saturation requirements are made only with respect to $A$. Therefore, the one-sided condition (4) in Corollary 3 may be considered more natural than the two-sided condition (12) in Corollary 6. Note in this connection that Hall's Theorem is not immediate from Corollary 6 .

\section{SOLUTION OF THE PROBLEM WITHOUT THE USE OF $k$-FACTORS}

From Theorem 4 we reprove Corollary 3 without the use of $k$-factors. As the first proof of Corollary 3 , this proof implies a (flow) algorithm that decides whether and how a collection of $k$ pairwise disjoint $A$-saturating matchings in a bipartite graph $G=(A, B)$ can be found.

Alternative Proof of Corollary 3. Call a spanning subgraph $H$ of $G k$-suitable if $d_{H}(x)=k$ for all $x \in A$ and $d_{H}(y) \leq k$ for all $y \in B$. We establish two claims:

(13) $G$ contains a $k$-suitable spanning subgraph if and only if (4) holds for all $X \subseteq A$.

Set

$$
\begin{aligned}
& f_{1}(v)=k \\
& f_{2}(v)=\left\{\begin{array}{ll}
d(v)-k & \text { for all } v \in V(G), \\
d(v) & \text { if } v \in A
\end{array},\right. \\
& e_{1}=e_{2}=e .
\end{aligned}
$$

Then, two spanning subgraphs $G_{1}=\left(A, B ; E_{1}\right)$ and $G_{2}$ $=\left(A, B ; E_{2}\right)$ of $G$ with $E_{1} \cup E_{2}=E$ and $E_{1} \cap E_{2}=\varnothing$ satisfy (6) if and only if $G_{1}$ is $k$-suitable. Furthermore, (7)-(10) hold if and only if (4) holds. Thus, (13) follows from Theorem 4 .

(14) The edge set of a $k$-suitable spanning subgraph of $G$ can be partitioned into the edge set of a $(k-1)$ suitable spanning subgraph and an $A$-saturating matching.

Let $H$ be a $k$-suitable spanning subgraph of $G$ and set

$$
\begin{array}{ll}
h_{1}(v)=1 & \text { for all } v \in V(H), \\
h_{2}(v)= \begin{cases}k-1 & \text { if } v \in A \\
\min \left\{k-1, d_{H}(v)\right\} & \text { if } v \in B\end{cases}
\end{array}
$$

Let $X \subseteq A$. Clearly, since $H$ is $k$-suitable, $\left|N_{H}(X)\right| \geq$ $|X|$. Hence,

$$
\begin{aligned}
\sum_{y \in B} \min \left\{h_{1}(y), q_{H}(X, y)\right\} & =\left|N_{H}(X)\right| \\
& \geq|X|=k|X|-(k-1)|X| \\
& =\sum_{x \in X}\left(d_{H}(x)-h_{2}(x)\right) .
\end{aligned}
$$

Set $B_{1}=\left\{y \in B \mid q_{H}(X, y)=k\right\}$ and $B_{2}=B-B_{1}$. Since $H$ is $k$-suitable, $\left|B_{1}\right| \leq|X|$. Hence,

$$
\begin{aligned}
\sum_{y \in B} \min & \left\{h_{2}(y), q_{H}(X, y)\right\} \\
& =(k-1)\left|B_{1}\right|+\sum_{y \in B_{2}} q_{H}(X, y) \\
& =(k-1)\left|B_{1}\right|+q_{H}\left(X, B_{2}\right) \\
& =(k-1)\left|B_{1}\right|+q_{H}(X, B)-q_{H}\left(X, B_{1}\right) \\
& =(k-1)\left|B_{1}\right|+k|X|-k\left|B_{1}\right|=k|X|-\left|B_{1}\right| \\
& \geq k|X|-|X|=\sum_{x \in X}\left(d_{H}(x)-h_{1}(x)\right) .
\end{aligned}
$$

By Theorem 4, (15) and (16) imply the existence of two spanning subgraphs $H_{1}$ and $H_{2}$ of $H$ such that

$$
\begin{aligned}
& E\left(H_{1}\right) \cup E\left(H_{2}\right)=E(H), E\left(H_{1}\right) \cap E\left(H_{2}\right)=\varnothing, \\
& d_{H_{i}}(v) \leq h_{i}(v) \quad(v \in V(H), i=1,2) .
\end{aligned}
$$


$H_{2}$ is $(k-1)$-suitable and $E\left(H_{1}\right)$ is an $A$-saturating matching, proving (14). Corollary 3 follows from (13) and (14) via induction on $k$.

We note that (14) could have been deduced immediately from the fact that a $k$-suitable subgraph of $G$ is $k$ edge-colorable. We chose to use Theorem 4 since it is applicable in more general contexts also. For example, our second proof of Corollary 3 is easily extended to a proof of the following result, where an A-saturating sclaw-factor of $G=(A, B)$ is defined as the edge set of a spanning subgraph $H$ of $G$ such that $d_{H}(x)=s$ for all $x \in A$ and $d_{H}(y) \leq 1$ for all $y \in B$.

Theorem 7. Let $G=(A, B)$ be a bipartite graph and $k$, s nonnegative integers. Then, $G$ has $k$ pairwise disjoint $A$-saturating $s$-claw-factors if and only if, for all $X \subseteq$ $A$,

$$
\sum_{y \in B} \min \{k s, q(X, y)\} \geq k s|X|
$$

\section{REFERENCES}

[1] J. A. Bondy and U. S. R. Murty, Graph Theory with Applications. Macmillan, London, Elsevier, New York (1976).

[2] J. Folkman and D. R. Fulkerson, Edge colorings in bipartite graphs. Combinatorial Mathematics and Its Applications. (R. C. Bose and T. A. Dowling, Eds.). University of North Carolina Press, Chapel Hill (1969) 561-577.

[3] L. R. Ford and D. R. Fulkerson, Flows in Networks. Princeton University Press, Princeton, NJ (1962).

[4] P. Katerinis, Minimum degree of bipartite graphs and the existence of $k$-factors. Graphs Combinatorics 6 (1990) 253-258.

[5] L. Lovász and M. D. Plummer, Matching theory. Ann. of Discrete Math. 29 (1986).

[6] E. A. van Doorn, personal communication.

Received July 1991

Accepted October 1992 